\title{
Internal Categories in Crossed Semimodules and Schreier Internal Categories
}

\author{
Sedat Temel*
}

\begin{abstract}
In this paper, we characterize internal categories in the category of crossed semimodules and the category of Schreier internal categories within monoids. Then we prove a natural equivalence between their categories. This allows us to produce various examples of double categories.

Keywords: Crossed module; Crossed semimodule; Schreier internal category; Double category.

AMS Subject Classification (2020): Primary: 20L05; Secondary: 18D35; 18B40; 20 M50.

${ }^{*}$ Corresponding author
\end{abstract}

\section{Introduction}

Crossed modules, which are defined by Whitehead in $[22,23]$ as algebraic models for homotopy 2-types, have been used with several branches of mathematics such as homotopy theory [6], homological algebra [10], and algebraic K-theory [12]. The structure of a crossed module $(A, B, \partial)$ has a pair of groups and a homomorphism $\partial$ with an action $\bullet$ of $B$ on $A$ satisfying $\partial(b \bullet a)=b \partial(a) b^{-1}$ and $\partial(a) \bullet a^{\prime}=a a^{\prime} a^{-1}$. The category of crossed modules is naturally equivalent to several algebraic and combinatorial categories such as group-groupoids (or alternatively called in literature as $\mathcal{G}$-groupoids [5], geoup-groupoids [7] or 2-groups [2]). This equivalence has been generalized in different ways: for example, by taking 2-groupoids with a single object [1]. The other one is the generalization to monoids by taking special types of internal categories like Schreier internal categories in the category MON of monoids using crossed semimodules by Patchkoria [15]. For the topological aspect of the results of [15], see [19]. For the 2-categorical approach to Schreier internal categories in MON using Schreier 2-categories with one object, see [20]. Patchkoria also defined the category of Schreier internal groupoids in MON which is equivalent to the category of crossed semimodules $C=(A, B, \partial)$ where A is a group. This natural equivalence is the special case of the main theorem of [16] by Porter.

Double groupoids, which should be thought of as internal groupoids in the category GPD of groupoids, were introduced by Ehresmann in $[8,9]$ and have been used in mathematics and mathematical physics as an application of categorical methods for some problems. As an example, one can see the reference [6] for the proof of the 2-dimensional Seifert-Van-Kampen Theorem using double groupoids. For an extension of topological quantum field theories via double categories, see [11]. Brown and Spencer proved the categorical equivalence between crossed modules and special double groupoids in the sense that the vertical and horizontal groupoids agree with a single point [4]. Using this equivalence, normal and quotient objects in the category of crossed modules over groupoids and of double groupoids with thin structures were compared and the corresponding structures related double groupoids were characterized in [14] (see also [21]).

In this paper, we characterize internal categories in the category of crossed semimodules which are extensions of internal categories in the category of crossed modules [17] and the category of Schreier internal categories in the category MON of monoids which are general cases of double group-groupoids characterized in [18]. Finally, we prove the categorical equivalence between their structures. This equivalence enables us to obtain more examples of double categories.

Received : 21-02-2020, Accepted : 17-04-2020 


\section{Preliminaries}

A category $\mathcal{C}=\left(C_{0}, C\right)$ consists of the set $C_{0}$ of objects, the set $C=\cup_{x, y \in C_{0}} C(x, y)$ of morphisms where $C(x, y)$ is the set of morphisms from $x$ to $y$ with the source and the target maps $s, t: C \rightarrow C_{0}$, respectively, such that $s(c)=x, t(c)=y$ where $x \stackrel{c}{\longrightarrow} y$, the associative composition map $m: C(y, z) \times C(x, y) \rightarrow C(x, z)$ usually written as $m(d, c)=d \circ c$ and the unit map $\varepsilon: C_{0} \rightarrow C, \varepsilon(x)=1_{x} \in C(x, x)$ such that $s \varepsilon=t \varepsilon=1_{C_{0}}, c \circ 1_{x}=c$ and $1_{x} \circ c^{\prime}=c^{\prime}$, where $s(c)=t\left(c^{\prime}\right)=x$. A groupoid $\mathcal{G}=\left(G_{0}, G\right)$ is a category in which all morphisms are invertible. For further details, see $[3,13]$.

Let $\mathcal{C}$ be a category and $D_{0}, D$ be two objects of $\mathcal{C}$ with maps $s, t, \varepsilon, m$ as morphisms of $\mathcal{C}$ such that satisfy the usual category axioms where $D_{s} \times_{t} D$ is the pullback of $s, t$ with $\pi_{1}, \pi_{2}$.

$$
D_{s} \times_{t} D \stackrel{m}{\longrightarrow} D \underset{t}{\stackrel{\frac{\varepsilon}{s}}{\rightleftarrows}} D_{0}
$$

Then $\mathcal{D}=\left(D_{0}, D\right)$ is called an internal category in $\mathcal{C}$, if the following conditions are satisfied:

IC 1. $s \varepsilon=t \varepsilon=1_{D_{0}}$;

IC 2. $s m=s \pi_{2}, t m=t \pi_{1}$;

IC 3. $m\left(1_{D} \times m\right)=m\left(m \times 1_{D}\right)$ and

IC 4. $m\left(\varepsilon s, 1_{D}\right)=m\left(1_{D}, \varepsilon t\right)=1_{D}$.

For further details, see [13].

Let $\mathcal{D}$ and $\mathcal{D}^{\prime}$ be two internal categories in $\mathcal{C}$. Then an internal functor $F=\left(f_{0}, f\right): \mathcal{D} \rightarrow \mathcal{D}^{\prime}$ consists of a pair of morphisms $f_{0}: D_{0} \rightarrow D_{0}^{\prime}$ and $f: D \rightarrow D^{\prime}$ in $\mathcal{C}$ such that

(i) $s^{\prime} f=f_{0} s, t^{\prime} f=f_{0} t$,

(ii) $\varepsilon^{\prime} f_{0}=f_{1} \varepsilon$,

(iii) $m^{\prime}(f \times f)=f m$.

Thus we can construct the category of internal categories in an arbitrary category $\mathcal{C}$ with pullbacks where the morphisms are internal functors (or morphisms of internal categories) as defined above. This category is denoted by $\operatorname{CAT}(\mathcal{C})$.

Let $\mathcal{M}=\left(M_{0}, M\right)$ be an internal category in the category MON of monoids. If for any $c \in M$ there exists a unique $\widehat{c} \in \operatorname{ker} s$ such that

$$
c=\widehat{c} \cdot \varepsilon s(c),
$$

then $\mathcal{M}$ is called a Schreier internal category in MON and this condition is called the Schreier condition [15]. In a Schreier internal category, the monoid multiplication and the composition of morphisms give the following interchange rule

$$
(d \circ c) \cdot\left(d^{\prime} \circ c^{\prime}\right)=\left(d \cdot d^{\prime}\right) \circ\left(c \cdot c^{\prime}\right)
$$

whenever compositions are defined. Using this rule, the composition of morphisms can be written in terms of the monoid product as

$$
d \circ c=\widehat{d} \cdot \widehat{c} \cdot \varepsilon s(c)
$$

for all $c, d \in M$ with $t(c)=s(d)$. This means that $\widehat{d \circ c}=\widehat{d} \cdot \widehat{c}$.

A Schreier internal groupoid in MON is a Schreier internal category in which all morphisms are invertible.

Let $\mathcal{M}, \mathcal{N}$ be Schreier internal categories in MON. An internal functor $F=\left(f_{0}, f\right): \mathcal{M} \rightarrow \mathcal{N}$ such that $f_{0}: M_{0} \rightarrow$ $N_{0}, f: M \rightarrow N$ are morphisms of monoids is called a morphism of Schreier internal categories in MoN.

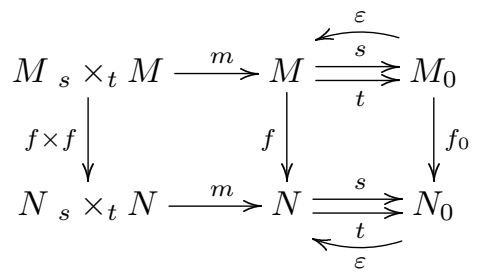


Hence Schreier internal categories in MON form a category which we denote by SIC.

A double category denoted by $\mathfrak{C}=(S, H, V, P)$ consists of the sets $S, H, V$ and $P$ of squares, horizontal and vertical morphisms (or edges) and points, respectively. The structure of a double category contains four compatible category structures as partially shown in the following diagram:

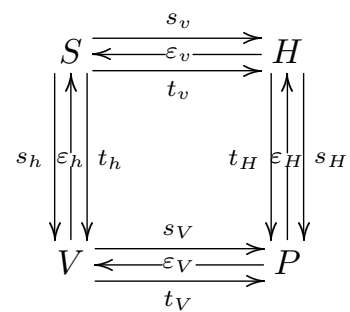

In a double category, vertical and horizontal morphisms can be composed as in an ordinary category, and squares can be composed vertically and horizontally.
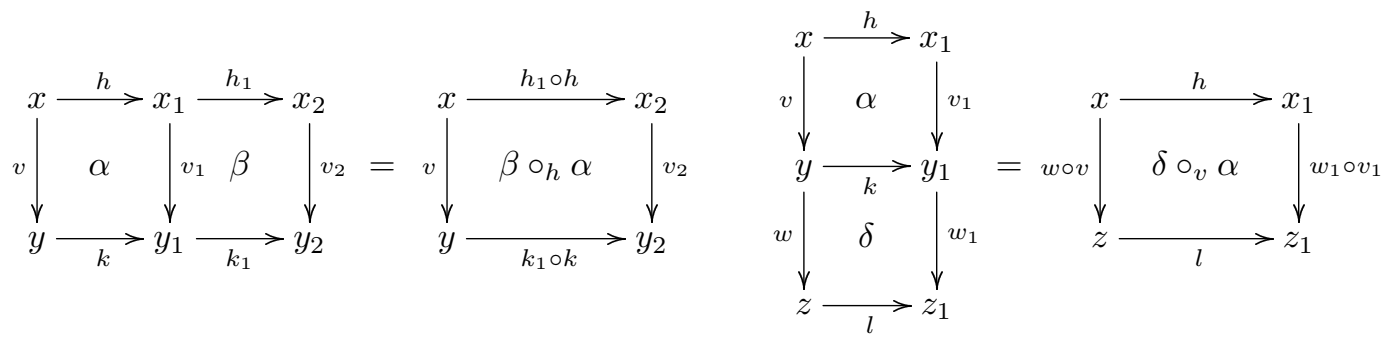

Horizontal composition and vertical composition of squares must satisfy the following interchange rule:

$$
\left(\theta \circ_{h} \delta\right) \circ_{v}\left(\beta \circ_{h} \alpha\right)=\left(\theta \circ_{v} \alpha\right) \circ_{h}\left(\delta \circ_{v} \alpha\right) .
$$

A double category can be regarded as an internal category in the category CAT of all small categories as shown in the following diagram:

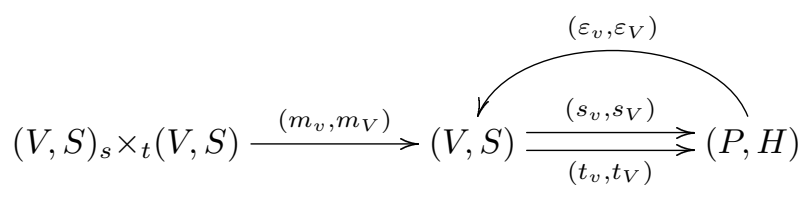

where $s=\left(s_{v}, s_{V}\right), t=\left(t_{v}, t_{V}\right)$. A double groupoid is a double category in which four underlying categories are groupoids. For further details, see $[4,8,9]$.

A crossed semimodule $C=(A, B, \partial)$ consists of a pair of monoids $A, B$ and a morphism $\partial: A \rightarrow B$ of monoids with an action $\bullet: B \times A \rightarrow A$ of $B$ on $A$ satisfying

CSM 1. $\partial(b \bullet a) \cdot b=b \cdot \partial(a)$

CSM 2. $\left(\partial(a) \bullet a_{1}\right) \cdot a=a \cdot a_{1}$,

for $a, a_{1} \in A$ and $b \in B$ [15].

Let $C=(A, B, \partial)$ and $C^{\prime}=\left(A^{\prime}, B^{\prime}, \partial^{\prime}\right)$ be crossed semimodules. A morphism of crossed semimodules is a mapping $f=\left\langle f_{1}, f_{2}\right\rangle: C \rightarrow C^{\prime}$ where $f_{1}: A \rightarrow A^{\prime}$ and $f_{2}: B \rightarrow B^{\prime}$ are morphisms of monoids such that $f_{1}(b \bullet a)=f_{2}(b) \bullet f_{1}(a)$ and $f_{2} \partial=\partial^{\prime} f_{1}$.

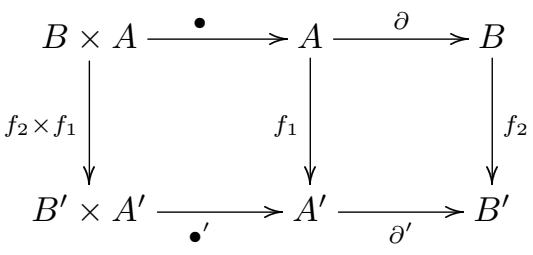

Hence crossed semimodules and their morphisms form a category which we will denote by CSM.

The following theorem was proved by Patchkoria in [15]. Since we need some details of the proof, we give a sketch proof in terms of our notation. 
Theorem 2.1. The category SIC of Schreier internal categories in MON is naturally equivalent to the category CSM of crossed semimodules.

Proof: A functor $\delta$ : SIC $\rightarrow$ CSM is defined as an equivalence of categories. Let $\mathcal{M}=\left(M_{0}, M\right)$ be a Schreier internal category in MON. Then $\delta(\mathcal{M})=(A, B, \partial)$ is a crossed semimodule where $A=\operatorname{ker} s, B=M_{0}, \partial=\left.t\right|_{\text {ker } s}$ and action of $M_{0}$ on ker $s$ is defined by $(x \bullet c) \cdot \varepsilon s(c)=\varepsilon s(c) \cdot c$ for all $x \in M_{0}$ and $c \in \operatorname{ker} s$.

A functor $\gamma:$ CSM $\rightarrow$ SIC is defined as a weak inverse of $\delta$. Let $C=(A, B, \partial)$ be a crossed semimodule. Then $\gamma(A, B, \partial)=(B, B \ltimes A, s, t, \varepsilon, \circ)$ is a Schreier internal category in MoN where $B \ltimes A$ is the semi-direct product of monoids with the product $(b, a) \cdot\left(b^{\prime}, a^{\prime}\right)=\left(b \cdot b^{\prime}, a \cdot\left(b \bullet a^{\prime}\right)\right)$. The structure maps are defined by $s(b, a)=b$, $t(b, a)=\partial(a) \cdot b, \varepsilon(b)=\left(b, e_{A}\right)$ where $e_{A}$ is the identity element of $A$ and the composition of morphisms is defined by $\left(\partial(a) \cdot b, a_{1}\right) \circ(b, a)=\left(b, a_{1} \cdot a\right)$. Since $(b, a)=\left(e_{B}, a\right) \cdot\left(b, e_{A}\right)$, all morphisms satisfy the Schreier condition where $e_{B}$ is the identity element of $B$.

In order to define a natural transformation $\eta: \mathcal{M} \rightarrow \gamma \delta(\mathcal{M})$, a map $\eta_{\mathcal{M}}$ is defined to be identity on objects, and is defined by $\eta_{\mathcal{M}}(c)=(s(c), \widehat{c})$ for $c \in M$.

In order to define a natural transformation $\mu: 1_{\mathrm{CSM}} \rightarrow \delta \gamma$, a map $\mu_{C}$ is defined to be identity on $B$ and is defined by $a \mapsto\left(e_{B}, a\right)$ on $A$.

The following results can be obtained as restrictions of this equivalence.

Corollary 2.1. [15] The category SIG of Schreier internal groupoids in MON is equivalent to the category of crossed semimodules $(A, B, \partial)$ where $A$ is a group.

Theorem 2.2. [5] The category of group-groupoids is equivalent to the category of crossed modules over groups.

\section{Internal categories in crossed semimodules}

Some properties of internal categories in the category CM of crossed modules are examined in [17]. In this section, we shall characterize internal categories in the category CSM of crossed semimodules and generalise some results given in [17]. Since the category MON of monoids has pullbacks then we can talk about internal categories in MON. Let $\mathcal{C}=\left(C_{0}, C, s, t, \varepsilon, m\right)$ be an internal category in CSM. This means that $\mathcal{C}$ consists of a pair of crossed semimodules $C_{0}=\left(A_{0}, B_{0}, \partial_{0}\right), C=(A, B, \partial)$ and four morphisms of crossed semimodules $s=\left\langle s_{A}, s_{B}\right\rangle$, $t=\left\langle t_{A}, t_{B}\right\rangle, \varepsilon=\left\langle\varepsilon_{A}, \varepsilon_{B}\right\rangle, m=\left\langle m_{A}, m_{B}\right\rangle$ which are called the source and the target maps, the identity object map and the composition map, respectively, as shown in the following diagram:

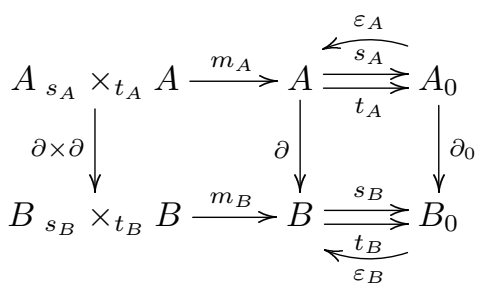

Since $s \varepsilon=\left\langle s_{A}, s_{B}\right\rangle\left\langle\varepsilon_{A}, \varepsilon_{B}\right\rangle=\left\langle s_{A} \varepsilon_{A}, \varepsilon_{B} s_{B}\right\rangle=\left\langle 1_{A_{0}}, 1_{B_{0}}\right\rangle=1_{C_{0}}$, the condition [IC 1] is satisfied. The other conditions [IC 1]-[IC 4] can be proved using a similar way.

Example 3.1. Let $C_{0}=(A, B, \partial)$ be a crossed semimodule. Since $C=(A \times A, B \times B, \partial \times \partial)$ is also a crossed semimodule where the action of $B \times B$ on $A \times A$ is defined by $\left(b, b^{\prime}\right) \bullet\left(a, a^{\prime}\right)=\left(b \bullet a, b^{\prime} \bullet a^{\prime}\right)$, then $\mathcal{C}=\left(C_{0}, C, s, t, \varepsilon, m\right)$ becomes an internal category in CSM where the structure maps are defined by $s=\left\langle\pi_{1}, \pi_{1}\right\rangle, t=\left\langle\pi_{2}, \pi_{2}\right\rangle, \varepsilon_{A}(a)=$ $(a, a), \varepsilon_{B}=(b, b)$ and $\left(a^{\prime}, a^{\prime \prime}\right) \circ\left(a, a^{\prime}\right)=\left(a, a^{\prime \prime}\right),\left(b^{\prime}, b^{\prime \prime}\right) \circ\left(b, b^{\prime}\right)=\left(b, b^{\prime \prime}\right)$, for $a, a^{\prime}, a^{\prime \prime} \in A, b, b^{\prime}, b^{\prime \prime} \in B$.

Example 3.2. Let $C=(A, B, \partial)$ be a crossed semimodule. Then $\mathcal{C}=(C, C, s, t, \varepsilon, m)$ is an internal category in CSM where the structure maps are identities.

Lemma 3.1. Let $\mathcal{C}=\left(C_{0}, C, s, t, \varepsilon, m\right)$ be an internal category in CSM. Then

(i) $s_{A}\left(a a^{\prime}\right)=s_{A}(a) s_{A}\left(a^{\prime}\right), t_{A}\left(a a^{\prime}\right)=t_{A}(a) t_{A}\left(a^{\prime}\right), s_{B}\left(b b^{\prime}\right)=s_{B}(b) s_{B}\left(b^{\prime}\right), t_{B}\left(b b^{\prime}\right)=t_{B}(b) t_{B}\left(b^{\prime}\right)$,

(ii) $\varepsilon_{A}\left(x x^{\prime}\right)=\varepsilon_{A}(x) \varepsilon\left(x^{\prime}\right), \varepsilon_{B}\left(y y^{\prime}\right)=\varepsilon_{B}(y) \varepsilon_{B}\left(y^{\prime}\right)$,

(iii) $\partial_{0} s_{A}=s_{B} \partial, \partial_{0} t_{A}=t_{B} \partial, \partial \varepsilon_{A}=\varepsilon_{B} \partial_{0}$, 
(iv) $s_{A}(b \bullet a)=s_{B}(b) \bullet s_{A}(a), t_{A}(b \bullet a)=t_{B}(b) \bullet t_{A}(a), \varepsilon_{A}(y \bullet x)=\varepsilon_{B}(y) \bullet \varepsilon_{A}(x)$,

for $a, a^{\prime} \in A, x, x^{\prime} \in A_{0}, b, b^{\prime} \in B, y, y^{\prime} \in B_{0}$.

Proof: The proof is clear, since $s=\left\langle s_{A}, s_{B}\right\rangle, t=\left\langle t_{A}, t_{B}\right\rangle, \varepsilon=\left\langle\varepsilon_{A}, \varepsilon_{B}\right\rangle$ are morphisms of crossed semimodules.

Lemma 3.2. Let $\mathcal{C}=\left(C_{0}, C, s, t, \varepsilon, m\right)$ be an internal category in CSM. Then

(i) $\left(a_{1} a_{1}^{\prime}\right) \circ\left(a a^{\prime}\right)=\left(a_{1} \circ a\right)\left(a_{1}^{\prime} \circ a^{\prime}\right),\left(b_{1} b_{1}^{\prime}\right) \circ\left(b b^{\prime}\right)=\left(b_{1} \circ b\right)\left(b_{1}^{\prime} \circ b^{\prime}\right)$

(ii) $\left(b_{1} \circ b\right) \bullet\left(a_{1} \circ a\right)=\left(b_{1} \bullet a_{1}\right) \circ(b \bullet a)$,

(iii) $\partial m_{A}=m_{B}(\partial \times \partial)$,

for $a, a^{\prime}, a_{1}, a_{1}^{\prime} \in A, b, b^{\prime}, b_{1}, b_{1}^{\prime} \in B$ such that $s_{A}\left(a_{1}\right)=t_{A}(a), s_{A}\left(a_{1}^{\prime}\right)=t_{A}\left(a^{\prime}\right), s_{B}\left(b_{1}\right)=t_{B}(b)$ and $s_{B}\left(b_{1}^{\prime}\right)=t_{B}\left(b^{\prime}\right)$.

Proof: The proof is clear, since $m=\left\langle m_{A}, m_{B}\right\rangle$ is a morphism of crossed semimodules.

The conditions (i)-(ii) are called interchange rules.

Definition 3.1. Let $\mathcal{C}$ and $\mathcal{C}^{\prime}$ be two internal categories in CSM. An internal functor $F=(f, g): \mathcal{C} \rightarrow \mathcal{C}^{\prime}$ is called morphism of internal categories in CSM, if $f_{0}: A_{0} \rightarrow A_{0}^{\prime}, f: A \rightarrow A^{\prime}, g_{0}: B_{0} \rightarrow B_{0}^{\prime}, f: B \rightarrow B^{\prime}$ such that $f=\left(f_{0}, f\right), g=\left(g_{0}, g\right)$ are functors and $\left\langle f_{0}, g_{0}\right\rangle,\langle f, g\rangle$ are crossed semimodule morphisms.

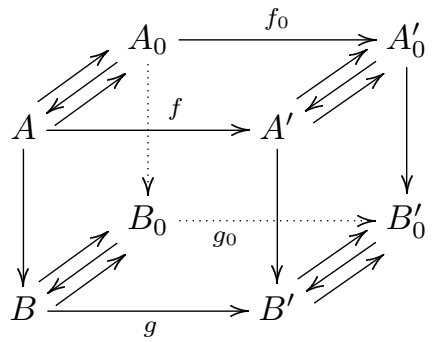

Hence we form the category of internal categories in CSM, which we denote by CAT(CSM).

\section{Internal categories in Schreier internal categories}

We know that double categories are internal categories in the category CAT of small categories. In this section, we characterize internal categories in the category SIC of Schreier internal categories in MON and generalize some results given in [18]. Let $\mathcal{K}=(\mathcal{M}, \mathcal{N}, s, t, \varepsilon, m)$ be an internal category in SIC. This means that $\mathcal{K}$ contains a pair of Schreier internal categories $\mathcal{M}=\left(M_{0}, M\right), \mathcal{N}=\left(N_{0}, N\right)$ in MoN and four morphisms $s=\left(s_{0}, s_{1}\right), t=\left(t_{0}, t_{1}\right), \varepsilon=\left(\varepsilon_{0}, \varepsilon_{1}\right)$ and $m=\left(m_{0}, m_{1}\right)$ of SIC as internal functors which are called the source and the target maps, the identity object map, and the composition map, respectively, as partially shown in the following diagrams:

$$
\left(M_{0}, M\right)_{s} \times_{t}\left(M_{0}, M\right) \stackrel{\left(m_{0}, m_{1}\right)}{\longrightarrow}\left(M_{0}, M\right) \stackrel{\left(\varepsilon_{0}, \varepsilon_{1}\right)}{\stackrel{\left(s_{0}, s_{1}\right)}{\longrightarrow}} \longrightarrow\left(N_{0}, N\right)
$$

Here the set of squares is $M$, the sets of horizontal and vertical morphisms are $N$ and $M_{0}$, respectively, and the set of points is $N_{0}$.

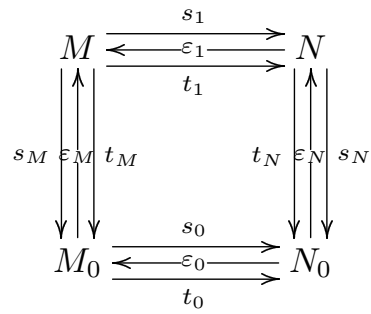


The composition $m_{M}$ is the horizontal composition of squares, and it will be denoted by $m_{h}$ or $\circ_{h}$ when no confusion arises. The composition $m_{1}$ of the category $\mathcal{M}$ is the vertical composition of squares, and it will be denoted by $m_{v}$ or $\circ_{v}$ when no confusion arises. Hence we replace $s_{1}, t_{1}, \varepsilon_{1}, s_{M}, t_{M}, \varepsilon_{M}$ by $s_{v}, t_{v}, \varepsilon_{v}, s_{h}, t_{h}, \varepsilon_{h}$, respectively. Due to Schreier condition, we write

$$
\alpha=\widehat{\alpha} \cdot \varepsilon_{h} s_{h}(\alpha), \quad n=\widehat{n} \cdot \varepsilon_{N} s_{N}(n)
$$

for $\alpha \in M, n \in N$. This structure denoted by $\mathfrak{C}=\left(M, N, M_{0}, N_{0}\right)$ is a generalization of the double group-groupoid concept, which is defined in [18].

Example 4.1. Given a Schreier internal category $\mathfrak{C}$ in $\mathrm{MON}$, then $\mathfrak{C}=\left(M, M_{0}, M, M_{0}\right)$ is an internal category in SIC with the trivial structural maps.

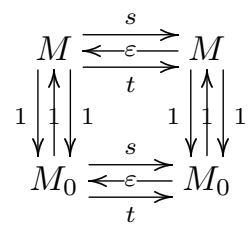

Lemma 4.1. Let $\mathfrak{C}=(S, H, V, P)$ be an internal category in SIC.

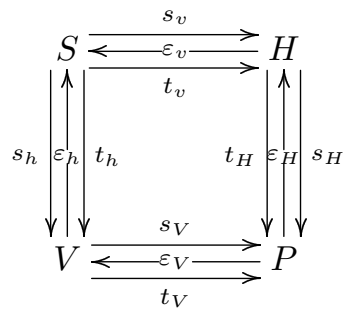

Then the following interchange rules are satisfied:

(i) $\left(\alpha_{4} \circ_{v} \alpha_{2}\right) \circ_{h}\left(\alpha_{3} \circ_{v} \alpha_{1}\right)=\left(\alpha_{4} \circ_{h} \alpha_{3}\right) \circ_{v}\left(\alpha_{2} \circ_{h} \alpha_{1}\right)$

(ii) $\left(\alpha_{2} \circ_{h} \alpha_{1}\right) \cdot\left(\alpha_{2}^{\prime} \circ_{h} \alpha_{1}^{\prime}\right)=\left(\alpha_{2} \cdot \alpha_{2}^{\prime}\right) \circ_{h}\left(\alpha_{1} \cdot \alpha_{1}^{\prime}\right)$

(iii) $\left(\alpha_{3} \circ_{v} \alpha_{1}\right) \cdot\left(\alpha_{3}^{\prime} \circ_{v} \alpha_{1}^{\prime}\right)=\left(\alpha_{3} \cdot \alpha_{3}^{\prime}\right) \circ_{v}\left(\alpha_{1} \cdot \alpha_{1}^{\prime}\right)$

whenever one side of the equations makes sense, for $\alpha_{1}, \alpha_{2}, \alpha_{3}, \alpha_{4} \in S$.

Lemma 4.2. Let $\mathfrak{C}=(S, H, V, P)$ be an internal category in SIC. Then the compositions of morphisms can be written in terms of the monoid operation as follows:

(i) $\beta \circ_{h} \alpha=\widehat{\beta} \cdot \widehat{\alpha} \cdot \varepsilon_{h} s_{h}(\alpha)$,

(ii) $k \circ h=\widehat{k} \cdot \widehat{h} \cdot \varepsilon_{H} s_{H}(h)$.

for $\alpha, \beta, \delta \in S$ and $k, h \in H$, whenever all compositions above are defined.

Definition 4.1. Let $\mathfrak{C}$ and $\mathfrak{C}^{\prime}$ be two internal categories in SIC. A morphism $F=\left(f_{s}, f_{h}, f_{v}, f_{p}\right): \mathfrak{C} \rightarrow \mathfrak{C}^{\prime}$ of double categories such that $f_{s}: S \rightarrow S^{\prime}, f_{h}: H \rightarrow H^{\prime}, f_{v}: V \rightarrow V^{\prime}$ and $f_{p}: P \rightarrow P^{\prime}$ are monoid homomorphisms is called morphism of internal categories in SIC.

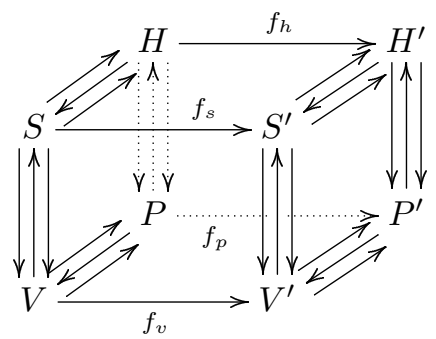


Hence we form the category of internal categories in SIC which we denote by CAT(SIC).

Theorem 4.1. The categories $\mathrm{CAT}(\mathrm{SIC})$ and $\mathrm{CAT}(\mathrm{CSM})$ are equivalent.

Proof: A functor $\delta: \operatorname{CAT}(\mathrm{SIC}) \rightarrow \mathrm{CAT}(\mathrm{CSM})$ is defined as in the following way. Let $\mathfrak{C}=(S, H, V, P)$ be an internal category in SIC. Then we obtain crossed semimodules $(A, B, \partial),\left(A_{0}, B_{0}, \partial_{0}\right)$ from Schreier internal categories $(V, S),(P, H)$, respectively, where $A=\operatorname{ker} s_{v}, B=V, \partial=\left.t_{v}\right|_{\operatorname{ker} s_{v}}$ and $A_{0}=\operatorname{ker} s_{H}, B=P, \partial_{0}=\left.t_{H}\right|_{\operatorname{ker} s_{H}}$.

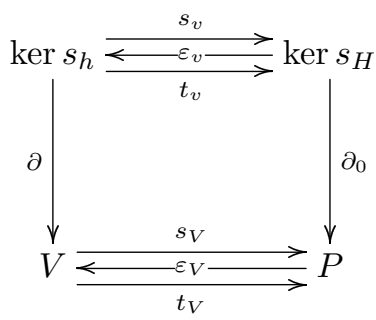

Here the action of $V$ on ker $s_{v}$ is defined by such that

$$
(v \bullet \alpha) \cdot \varepsilon_{h}(v)=\varepsilon_{h}(v) \cdot \alpha
$$

and the action of $P$ on $\operatorname{ker} s_{H}$ is defined by such that

$$
(p \bullet h) \cdot \varepsilon_{H}(p)=\varepsilon_{H}(p) \cdot h
$$

where $p \in P, h \in \operatorname{ker} s_{H}, v \in V$ and $\alpha \in \operatorname{ker} s_{h}$. We shall prove that $\left\langle s_{v}, s_{V}\right\rangle$ is a morphism of crossed semimodules. Clearly $s_{V} \partial=\partial_{0} s_{v}, t_{V} \partial=\partial_{0} t_{v}$ and $\partial \varepsilon_{v}=\varepsilon_{H} \partial_{0}$. Since $\varepsilon_{H} s_{V}=s_{v} \varepsilon_{h}$, we write

$$
\begin{aligned}
\left(s_{V}(v) \bullet s_{v}(\alpha)\right) \cdot \varepsilon_{H} s_{V}(v) & =\varepsilon_{H} s_{V}(v) \cdot s_{v}(\alpha) \\
& =s_{v} \varepsilon_{h}(v) \cdot s_{v}(\alpha) \\
& =s_{v}\left(\varepsilon_{h}(v) \cdot \alpha\right) \\
& =s_{v}\left((v \bullet \alpha) \cdot \varepsilon_{h}(v)\right) \\
& =s_{v}(v \bullet \alpha) \cdot s_{v} \varepsilon_{h}(v) \\
& =s_{v}(v \bullet \alpha) \cdot \varepsilon_{H} s_{V}(v) .
\end{aligned}
$$

Let $h=\left(s_{V}(v) \bullet s_{v}(\alpha)\right) \cdot \varepsilon_{H} s_{V}(v)$. Since $h \in H, s_{H} \varepsilon_{H}=1_{H}$ and $s_{V}(v) \bullet s_{v}(\alpha) \in \operatorname{ker} s_{H}$, we have

$$
\varepsilon_{H} s_{H}(h)=\varepsilon_{H} s_{H}\left(s_{V}(v) \bullet s_{v}(\alpha)\right) \cdot \varepsilon_{H} s_{H} \varepsilon_{H} s_{V}(v)=\varepsilon_{H} s_{V}(v) .
$$

Let $k=s_{v}(v \bullet \alpha) \cdot \varepsilon_{H} s_{V}(v)$. Similarly we get

$$
\varepsilon_{H} s_{H}(k)=\varepsilon_{H} s_{V}(v) .
$$

Therefore, under the Schreier condition, we have

$$
s_{v}(v \bullet \alpha)=s_{V}(v) \bullet s_{v}(\alpha) .
$$

Using a similar way, we get $\left\langle t_{v}, t_{V}\right\rangle$ and $\left\langle\varepsilon_{v}, \varepsilon_{V}\right\rangle$ are morphisms of crossed semimodules.

Let $F=\left(f_{s}, f_{h}, f_{v}, f_{p}\right):(S, H, V, P) \rightarrow\left(S^{\prime}, H^{\prime}, V^{\prime}, P^{\prime}\right)$ be a morphism of CAT(SIC). Then $\delta(F)=(f, g)$ is a morphism of CAT(CSM) where $f=\left(f_{h},\left.f_{s}\right|_{\operatorname{ker} s_{h}}\right), g=\left(f_{p},\left.f_{v}\right|_{\operatorname{ker} s_{H}}\right)$. We will only prove that $\left\langle\left. f_{s}\right|_{\operatorname{ker} s_{h}},\left.f_{v}\right|_{\operatorname{ker} s_{H}}\right\rangle$ is a morphism of crossed semimodules. Since $f_{s} \varepsilon_{h}=\varepsilon_{H} f_{v}$, we get

$$
\begin{aligned}
f_{s}(v \bullet \alpha) \cdot f_{s} \varepsilon_{h}(v) & =f_{s}\left((v \bullet \alpha) \cdot \varepsilon_{h}(v)\right) \\
& =f_{s}\left(\varepsilon_{h}(v) \cdot \alpha\right) \\
& =f_{s} \varepsilon_{h}(v) \cdot f_{s}(\alpha) \\
& =\varepsilon_{H} f_{v}(v) \cdot f_{s}(\alpha) \\
& =\left(f_{v}(v) \bullet f_{s}(\alpha)\right) \cdot \varepsilon_{H} f_{v}(v) \\
& =\left(f_{v}(v) \bullet f_{s}(\alpha)\right) \cdot f_{s} \varepsilon_{h}(v) .
\end{aligned}
$$


Let $\alpha^{\prime}=f_{s}(v \bullet \alpha) \cdot f_{s} \varepsilon_{h}(v)$. Since $\alpha^{\prime} \in S^{\prime}, s_{h}^{\prime} f_{s}=f_{v} s_{h}, \varepsilon_{h}^{\prime} f_{v}=f_{s} \varepsilon_{h}, s_{h} \varepsilon_{h}=1_{S}$ and $f_{s}(v \bullet \alpha) \in$ ker $s_{H}^{\prime}$, we have

$$
\varepsilon_{h}^{\prime} s_{h}^{\prime}\left(\alpha^{\prime}\right)=\varepsilon_{h}^{\prime} s_{h}^{\prime} f_{s}(v \bullet \alpha) \cdot \varepsilon_{h}^{\prime} s_{h}^{\prime} f_{s} \varepsilon_{h}(v)=\varepsilon_{h}^{\prime} f_{v} s_{h} \varepsilon_{h}(v)=\varepsilon_{h}^{\prime} f_{v}(v)=f_{s} \varepsilon_{h}(v) .
$$

Let $\beta^{\prime}=\left(f_{v}(v) \bullet f_{s}(\alpha)\right) \cdot f_{s} \varepsilon_{h}(v)$. Using a similar way, we get

$$
\varepsilon_{h}^{\prime} s_{h}^{\prime}\left(\beta^{\prime}\right)=f_{s} \varepsilon_{h}(v) .
$$

Hence, under the Schreier condition, we have

$$
f_{s}(v \bullet \alpha)=f_{v}(v) \bullet f_{s}(\alpha) .
$$

Now we define a functor $\gamma: \operatorname{CAT}(\mathrm{CSM}) \rightarrow \operatorname{CAT}(\mathrm{SIC})$ as a weak inverse of the functor $\delta$. Given an internal category $\mathcal{C}=\left(C_{0}, C, s, t, \varepsilon, m\right)$ in CSM where $C_{0}=\left(A_{0}, B_{0}, \partial_{0}\right), C=(A, B, \partial)$ are crossed semimodules and $s=\left\langle s_{A}, s_{B}\right\rangle, t=\left\langle t_{A}, t_{B}\right\rangle, \varepsilon=\left\langle\varepsilon_{A}, \varepsilon_{B}\right\rangle, m=\left\langle m_{A}, m_{B}\right\rangle$ are morphisms of crossed semimodules, we obtain Schreier internal categories $(B, B \ltimes A)$ and $\left(B_{0}, B_{0} \ltimes A_{0}\right)$ by the Theorem 2.1 as partially shown in the following diagram:

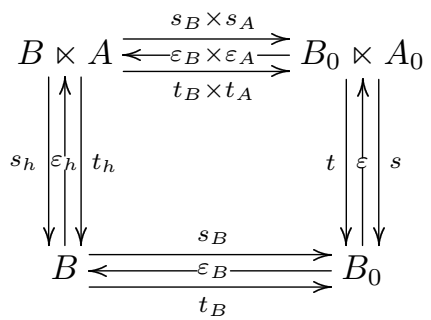

Here pairs $(b, a)$ are squares and $\left(b_{0}, a_{0}\right),\left(d_{0}, c_{0}\right)$ are horizontal morphisms as

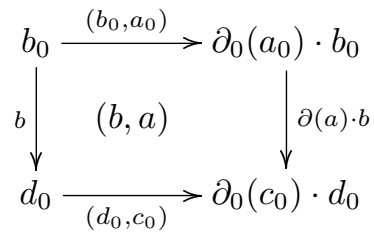

for $b_{0}, d_{0} \in B_{0}, b \in B, a_{0}, c_{0} \in A_{0}, a \in A$ where $a_{0} \stackrel{a}{\longrightarrow} c_{0}$. The horizontal composition of squares is defined by

$$
(\partial(a) \cdot b, c) \circ_{h}(b, a)=(b, c \cdot a)
$$

where

$$
\left(\partial_{0}\left(a_{0}\right) \cdot b_{0}, c_{0}\right) \circ\left(b_{0}, a_{0}\right)=\left(b_{0}, c_{0} \cdot a_{0}\right) .
$$

The vertical composition of squares is defined by

$$
(d, c) \circ_{v}(b, a)=(d \circ b, c \circ a)
$$

whenever $d \circ b, c \circ a$ are defined. The product of squares are defined by

$$
(b, a) \cdot\left(b^{\prime}, a^{\prime}\right)=\left(b \cdot b^{\prime}, a \cdot\left(b \bullet a^{\prime}\right)\right)
$$

where $b, b^{\prime} \in B, a, a^{\prime} \in A$. Since $(b, a)=\left(e_{B}, a\right) \cdot\left(b, e_{A}\right)$, all squares satisfy the Schreier condition.

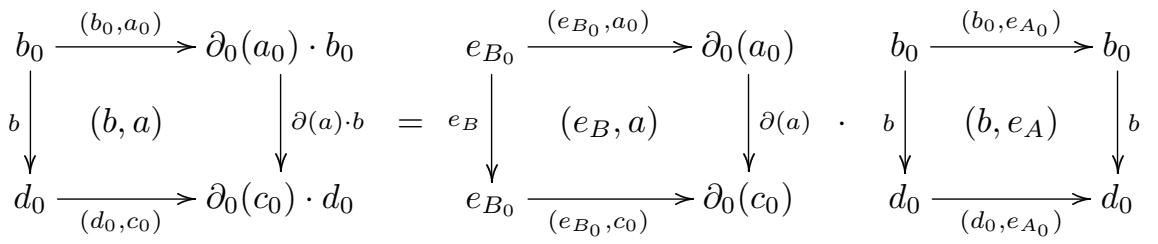

Let $F=(f, g)$ be a morphism of CAT(CSM) where $f=\left(f_{0}, f\right), g=\left(g_{0}, g\right)$. Then

$$
\gamma(F)=\left(g \times f, g_{0} \times f_{0}, g, g_{0}\right)
$$


is a morphism of $\mathrm{CAT}(\mathrm{CSM})$.

In order to prove $1_{\mathrm{CAT}(\mathrm{SIC})} \cong \gamma \delta$, we define a natural transformation

$$
\eta: \mathfrak{C} \rightarrow \gamma \delta(\mathfrak{C})
$$

through a map $\eta_{\mathfrak{C}}$ such that to be identity on points and on vertical morphisms,

$$
\eta_{\mathfrak{C}}(h)=\left(s_{H}(h), \widehat{h}\right), \quad \eta_{\mathfrak{C}}(\alpha)=\left(s_{h}(\alpha), \widehat{\alpha}\right),
$$

for $h \in H, \alpha \in S$. We will verify that $\eta_{\mathfrak{C}}$ preserves monoid multiplication and vertical and horizontal compositions.

$$
\begin{aligned}
\eta_{\mathfrak{C}}\left(\alpha \cdot \alpha^{\prime}\right) & =\eta_{\mathfrak{C}}\left(\widehat{\alpha} \cdot \varepsilon_{h} s_{h}(\alpha) \cdot \widehat{\alpha^{\prime}} \cdot \varepsilon_{h} s_{h}\left(\alpha^{\prime}\right)\right) \\
& =\eta_{\mathfrak{C}}\left(\widehat{\alpha} \cdot\left(s_{h}(\alpha) \bullet \widehat{\alpha^{\prime}}\right) \cdot \varepsilon_{h} s_{h}(\alpha) \cdot \varepsilon_{h} s_{h}\left(\alpha^{\prime}\right)\right) \\
& =\eta_{\mathfrak{C}}\left(\widehat{\alpha} \cdot\left(s_{h}(\alpha) \bullet \widehat{\alpha^{\prime}}\right) \cdot \varepsilon_{h} s_{h}\left(\alpha \cdot \alpha^{\prime}\right)\right) \\
& =\left(s_{h}\left(\alpha \cdot \alpha^{\prime}\right), \widehat{\alpha} \cdot\left(s_{h}(\alpha) \bullet \widehat{\alpha^{\prime}}\right)\right) \\
& =\left(s_{h}(\alpha) \cdot s_{h}\left(\alpha^{\prime}\right), \widehat{\alpha} \cdot\left(s_{h}(\alpha) \bullet \widehat{\alpha^{\prime}}\right)\right) \\
& =\left(s_{h}(\alpha), \widehat{\alpha}\right) \cdot\left(s_{h}\left(\alpha^{\prime}\right), \widehat{\alpha^{\prime}}\right) \\
& =\eta_{\mathfrak{C}}(\alpha) \cdot \eta_{\mathfrak{C}}\left(\alpha^{\prime}\right)
\end{aligned}
$$

for $\alpha, \alpha^{\prime} \in S$,

$$
\eta_{\mathfrak{C}}\left(\beta \circ_{h} \alpha\right)=\eta_{\mathfrak{C}}\left(\widehat{\beta} \cdot \widehat{\alpha} \cdot \varepsilon_{h} s_{h}(\alpha)\right)=\left(s_{h}(\alpha), \widehat{\beta} \cdot \widehat{\alpha}\right)=\left(s_{h}(\beta), \widehat{\beta}\right) \circ_{h}\left(s_{h}(\alpha), \widehat{\alpha}\right)=\eta_{\mathfrak{C}}(\beta) \circ_{h} \eta_{\mathfrak{C}}(\alpha)
$$

whenever $\beta \circ_{h} \alpha$ is defined for $\beta, \alpha \in S$. Since

$$
\delta \circ_{v} \alpha=\left(\widehat{\delta} \cdot \varepsilon_{h} s_{h}(\delta)\right) \circ_{v}\left(\widehat{\alpha} \cdot \varepsilon_{h} s_{h}(\alpha)\right)=\left(\widehat{\delta} \circ_{v} \widehat{\alpha}\right) \cdot\left(\varepsilon_{h} s_{h}(\delta) \circ_{v} \varepsilon_{h} s_{h}(\alpha)\right)=\left(\widehat{\delta} \circ_{v} \widehat{\alpha}\right) \cdot \varepsilon_{h} s_{h}\left(\delta \circ_{v} \alpha\right),
$$

under the Schreier condition, we can write

$$
\eta_{\mathfrak{C}}\left(\delta \circ_{v} \alpha\right)=\left(s_{h}(\delta) \circ s_{h}(\alpha), \widehat{\delta} \circ_{v} \widehat{\alpha}\right)=\left(s_{h}(\delta), \widehat{\delta}\right) \circ_{v}\left(s_{h}(\alpha), \widehat{\alpha}\right)=\eta_{\mathfrak{C}}(\delta) \circ_{v} \eta_{\mathfrak{C}}(\alpha) .
$$

In order to prove $1_{\mathrm{CAT}(\mathrm{CSM})} \cong \delta \gamma$, we define a natural transformation

$$
\mu: 1_{\mathrm{CAT}(\mathrm{CSM})} \rightarrow \delta \gamma,
$$

through a map $\mu_{\mathcal{C}}$ which is defined to be identity on $A_{0}$ and $B_{0}, \mu_{\mathcal{C}}(a)=\left(e_{B}, a\right)$ and $\mu_{\mathcal{C}}\left(a_{0}\right)=\left(e_{B_{0}}, a_{0}\right)$ for $a \in A, a_{0} \in A_{0}$.

Other details are straightforward, and so is omitted.

Then we can write the following corollary as a restriction of this theorem.

Corollary 4.1. The category of double group-groupoids is equivalent to the category of internal categories in the category $\mathrm{CM}$ of crossed modules.

\section{References}

[1] Baez, J.C., Baratin, A., Freidel, L. and Wise, D.K.: Infinite-Dimensional Representations of 2-Groups. Mem. Am. Math. Soc. 219, (1032) (2012).

[2] Baez, J.C., Lauda, A.D.: Higher Dimensional Algebra V: 2-Groups. Theory Appl. Categ. 12, 423-491 (2004).

[3] Brown, R.: Topology and Groupoids. BookSurge LLC, North Carolina (2006).

[4] Brown, R, Spencer, C.B.: Double groupoids and crossed modules. Cahiers de Topologie et Géométrie Différentielle Catégoriques 17 (4), 343-362 (1976). 
[5] Brown, R., Spencer, C.B.: G-groupoids, crossed modules and the fundamental groupoid of a topological group. Mathematical Sciences and Applications E-Notes. Indagat. Math. 79 (4), 296-302 (1976).

[6] Brown, R., Higgins, P. J. and Sivera, R.: Nonabelian Algebraic Topology: Filtered spaces, crossed complexes, cubical homotopy groupoids. European Mathematical Society Tracts in Mathematics 15 (2011).

[7] Brown, R., Mucuk, O.: Covering groups of non-connected topological groups revisited. Math. Proc. Camb. Phil. Soc. 115, 97-110 (1994).

[8] Ehresmann, C.: Catégories doubles et catégories structurées. C. R. Acad. Sci. Paris 256, 1198-1201 (1963).

[9] Ehresmann, C.: Catégories structurées. Ann. Sci. Ec. Norm. Super. 80, 349-425 (1963b).

[10] Huebschmann, J.: Crossed n-fold extensions of groups and cohomology. Comment. Math. Helvetici. 55: 302-314 (1980).

[11] Kerler, T. and Lyubashenko, V.V.: Non-Semisimple Topological Quantum Field Theories for 3-Manifolds with Corners. Springer-Verlag. Berlin, Heidelberg, (2001).

[12] Loday, J.-L.: Cohomologie et groupes de Steinberg relatifs. J. Algebra. 54 178-202 (1978).

[13] Maclane, S.: Categories for the Working Mathematician, Graduate Text in Mathematics. 5, Springer-Verlag. New York (1971).

[14] Mucuk, O., Demir S.: Normality and quotient in crossed modules over groupoids and double groupoids. Turk J Math, 42, $2336-2347$ (2018).

[15] Patchkoria, A.: Crossed Semimodules and Schreier Internal Categories In The Category of Monoids. Georgian Math. J. 5(6), 575-581 (1998).

[16] Porter, T.: Crossed Modules in Cat and a Brown-Spencer Theorem for 2-Categories. Cah. Topol. Géom. Différ. Catég. XXVI-4 (1985).

[17] Şahan, T., Mohammed, J.J.: Categories internal to crossed modules Sakarya University Journal of Science. 23 (4), 519-531, (2019).

[18] Temel, S., Şahan, T. and Mucuk, O.: Crossed modules, double group-groupoids and crossed squares. Preprint arxiv:1802.03978v2 (2018).

[19] Temel, S.: Topological Crossed Semimodules and Schreier Internal Categories in the Category of Topological Monoids. Gazi University Journal of Science. 29 (4), 915-921 (2016).

[20] Temel, S.: Crossed semimodules of categories and Schreier 2-categories. Tbilisi Math. J. 11 (2), 47-57 (2018).

[21] Temel, S.: Normality and quotient in crossed modules over groupoids and 2-groupoids. Korean J. Math. 27 (1), 151-163 (2018).

[22] Whitehead, J.H.C.: Combinatorial homotopy II. Bull. Amer. Math. Soc. 55, 453-496 (1949).

[23] Whitehead, J.H.C.: Note on a previous paper entitled "On adding relations to homotopy group". Ann. Math. 47,806-810 (1946).

\section{Affiliations}

SEDAT TEMEL

ADDRESS: RecepTayyip Erdogan University, Dept. of Mathematics, 53100, Rize-Turkey.

E-MAIL: sedat.temel@erdogan.edu.tr

ORCID ID:0000-0001-6553-8758 